\title{
DEVELOPMENT OF A TOOL FOR VISUALIZING USER'S KANSEI ON THE WEB
}

\author{
Kun-Pyo LEE*, Chang-Min PARK** \\ *Department of Industrial Design, KAIST, 373-1, Kusong-dong, Yusung-gu, Taejon, KOREA \\ **YAHOO Korea, 648-26, Yoksam-Dong, Gangnam-Ku, Seoul, 135-080, KOREA
}

\begin{abstract}
Web has been widely used in everyday life and become necessary commodity, which makes web as major focus on user research. Users use WWW for diverse purposes. Some use the web for specific purpose and task but others are just surfing without any particular task in their mind. This ambiguous user's behavior is very difficult to understand. Particularly, among various difficulties, the most difficult part is that users do not show any concrete activities and they only calmly sit down with mouseclicking and eyeball movement. It becomes extremely hard to understand what users have in their mind. The paper develops and introduces the tool for visualizing user's tacit Kansei over the web by displaying mouse traces user left for using web. The mousetracking traces back all the paths users clicked on while he is navigating and visualizes them with displaying the time taken and stayed for clicks. It reveals user's Kansei on the web such as attention, preferences, navigating path and interests. The sample case is introduced for demonstrating mouse-tracking program and evaluating its validity.
\end{abstract}

Keywords: Mouse tracking, Web, Kansei

\section{INTRODUCTION}

Web has been increasingly becoming major channel for information and commodity tool of everyday life. This rapid expansion of web use has also brought up the expansion of range of users: some uses the web for specific purpose and tasks while others are just surfing without any explicit task in their mind. This diversity of users range and tasks has made far more difficult to conduct the user study of web.

Another factor which makes web user study more difficult is quiet behavior of users while using product. Unlike salient user's behavior of using physical product, users using web only shows the calm behavior of mouseclicking and unnoticeable eyeball movement. It has become extremely difficult for designer to tell what user has in his mind and what difficulties he is experiencing from observing his behavior. Besides, by definition, Kanse $i$ is a very subjective and unexplainable function. [1] Current major methods for user study of web like heuristics, questionnaire or interview cannot solve the problems because they are mainly relying on user's or expert's opinion. Such opinion-based methods cannot be of any help because opinions are only concerned with recognized problems. Many critical problems which routinely escape user's conscious awareness cannot be restated by users. In addition, the usability in the web is different from conventional usability of software and digital information appliances. In product design and software design, users pay first and experience usability later while on the web, users experience usability first and pay later. [2]

Based on the problems mentioned above, the present study sets the goal of this study as to develop the tool for understanding user's tacit Kansei over the web by visualizing through software developed for tracking mouse traces user left while he is using web. The study also reviews current methods for user study in the web and identifies the problems which, in turn, are used as base for development of new method for understanding user's Kansei in web. More specific objectives are as follows:

-To review current methods of user study in the web.

-To develop the tool which can reveal user's Kansei on the web.

-To explore the possibilities of applying the program for understanding user's Kansei on the web.

\section{UNDERSTANTING USER'S KANSEI ON WEB AND ITS PROBEMS}

There are wide varieties of methods available for user 
study in the web, many of which are borrowed from usability study methods originally developed for physical product or software. They include interview, questionnaire and survey, focus group interview, heuristic evaluation, cognitive walkthrough, guideline checklist, GOMS, task analysis, user testing, and prototyping. [3] Recently some more direct methods are introduced for web usability study. They include ethnographic study, filed observation, log file analysis, and eye tracking. These methods can be categorized into three groups as shown in Table 1 .

Table 1: Categories of methods for user study in the web and their problems

\begin{tabular}{l|l|l}
\hline Category & Methods & Problems \\
\hline Inquiry & $\begin{array}{l}\text { Interview, Questionnaire, } \\
\text { Survey, Focus group }\end{array}$ & $\begin{array}{l}\text { Hardly finds } \\
\text { real problems } \\
\text { users are } \\
\text { unaware of }\end{array}$ \\
\hline Inspection & $\begin{array}{l}\text { Heuristic evaluation, } \\
\text { Cognitive walkthrough, } \\
\text { Guideline checklist, } \\
\text { GOMS, Task analysis, } \\
\text { Prototyping }\end{array}$ & $\begin{array}{l}\text { Rely on only } \\
\text { experts' } \\
\text { opinion. }\end{array}$ \\
\hline $\begin{array}{l}\text { User } \\
\text { observation }\end{array}$ & $\begin{array}{l}\text { Ethnographic study, Field } \\
\text { observation, Log file } \\
\text { analysis, User testing, Eye } \\
\text { tracking }\end{array}$ & $\begin{array}{l}\text { Time, labor, } \\
\text { cost. }\end{array}$ \\
\hline
\end{tabular}

The first group is 'inquiry' group where designers ask users their opinions regarding web: interview, questionnaire and survey, and focus group interview. This inquiry method is easiest to implement and due to its simplicity, it is most widely used. However, this category of inquiring method has difficulty in finding critical problems which user is not even aware. User can hardly remember all the problems and interests he experienced through complex navigation. Even for problems user recognizes, it is very hard for him to elaborate them.

The second category is 'inspection' where experts inspect the web usability according to guidelines or checklists: heuristic evaluation, cognitive walkthrough, GOMS, task analysis and prototyping. This category of inspection methods is also useful in terms of its relative simplicity and economy. However experts are not necessarily to represent real users. Problems to real users may not be problems to experts or vice versa.

The last category is 'user observation' in which user directly participates the testing or just plainly navigating web without any intervention of designer: user testing, ethnographic testing, field observation, log file analysis, and eye tracking. Designer only observes or records what user is doing. This category of user observation method can solve two types of problems compared with two previous categories of methods: designer can get the data from real user's unbiased natural behavior. However there are still problems in implementing these methods. It generally requires lots of time, labor, and cost too. For example, to conduct ethnographic study, designer should collect many data from videotaped records or note-taking. Even he gathers all the data it is even harder to analyze all the data to identify meaningful findings: rewinding, forwarding, pausing, and annotating videotape is really annoying and time-consuming tasks. Eye tracking tracks all the traces where user's eyesight stayed over web navigating. Once this method is successfully implemented it generates really useful data because it shows quite natural human behavior. Besides user does not have any burden to remember and elaborate all his behavior while using web. It graphically shows all the eyesight traces. As shown in Figure 1, it reveals all the movement of eyesight and time stayed in specific area, which shows users preference and interests on the web page. However, in order to conduct the test, the user should harness rather heavy and cumbersome helmettype eye tracker and auxiliary equipment which prevents user from moving his head beyond the required calibration. Note user with strange outfit around his face shown in Figure 2. Due to this award equipment, user cannot generate natural behavior. In addition the equipment of eye tracker itself is rather expensive.

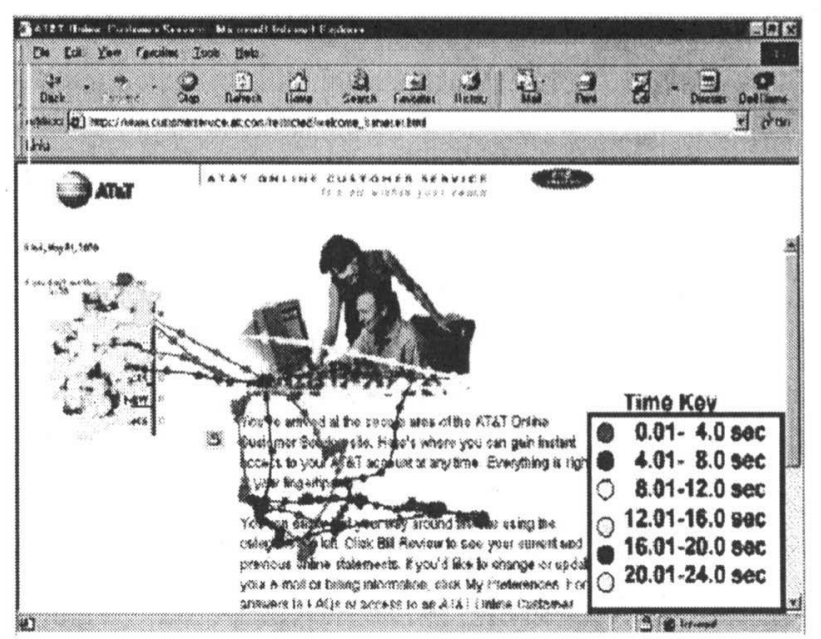

Figure 1: Sample screen showing eye movement of a user [4] 


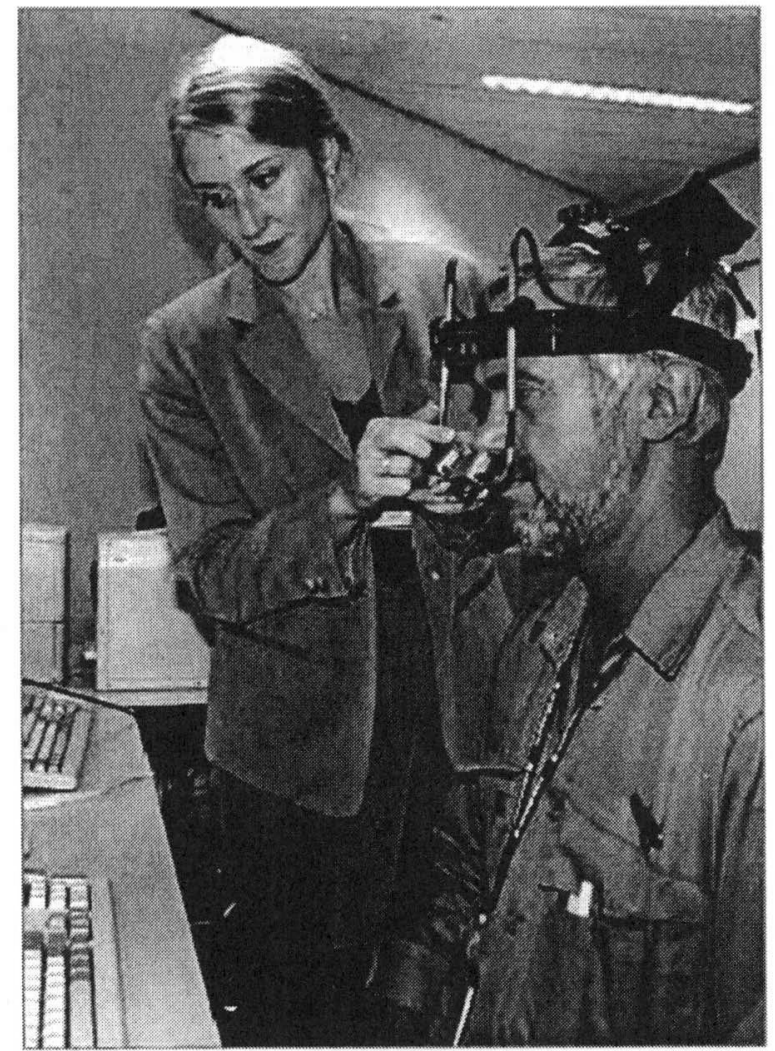

Figure 2: User participating the experiment using eye tracker [5]

\section{DEVELOPMENT OF THE TOOL FOR UNDERSTANDING USER'S KANSEI ON THE WEB}

Based on the problem found above, the tool for understanding user's perception on the web is proposed. The tool is developed based on the following principles:

- The tool should be used naturally as much as possible. The use of tool should not interfere user's natural interaction with web.

- The tool should be simple enough to cost least effort and time.

- The tool should be able to visualize users Kanse $i$ interests in using web.

- The tool should allow designer to collect and analyze the data very easily

- The tool should be integrated with other general purpose of usability tools.

3.1 Mouse as tool for understanding user's perceptual interest in using web

With very few exceptions, web is currently using mouse as input device. Almost all users are using mouse for moving around the web, clicking where he is interested in, scrolling down or up for viewing, or selecting necessary items from web. Mouse is almost standard input device for current web interface.

With this popularity of mouse as input device, mouse can be effective medium to track down user's interaction behavior with web. For example Barker and others proposed that user's mouse operation should be concerned to understand goodness of interface layout defining it as four steps: initiation period, sub-movement, pause, and verification time. [6] The study demonstrated user's mouse operation could be used to understand user's interaction behavior. In addition, mouse cost far less than other auxiliary equipments like eye tracker. User does not have to buy any independent equipment. Anyway users have to use mouse for using computer.

\subsection{Development of MouWow}

The program called MouWou is developed for tracking down user's mouse operation, visualizing its operational path, and analyzing the data in various aspects. MouWow consists of two modules: one is 'Mouse Tracker' for tracking down all the mouse operation and displaying all the paths users clicked and moved around; the other one is 'Mouse Analyzer' for saving all the web pages in bitmap with visualized mouse traces on it and allowing designer to perform various types of analysis like sorting, searching for specific pages, or statistical treatment. Each of module is introduced in the following section.

\subsection{Mouse Tracker}

Mouse Tracker records the entire paths user navigated over web pages with time taken and stayed. It also visualizes all the results and replay all the mouse movement.

When Mouse Tracker is started first, very simple small menu widow shown in the top of Figure 3 is activated. It has the function of making new file or saving file where all the records are saved, recording mouse operation, resetting current operation, transferring to 'Quick Viewer' mode for visualizing mouse operations. If user wants to record his mouse operation he can start by simple clicking 'Record' button. Once record is started, all the data such as duration time, sequence of operation are recorded in the system memory. In addition, screen image of web page is also saved in bitmap file whenever user changes the web page. The sub-menus of "File' and "Config" are shown in the bottom part of Figure 3. As shown in the Figure 3, sub-menus have diverse options user can choose from. 


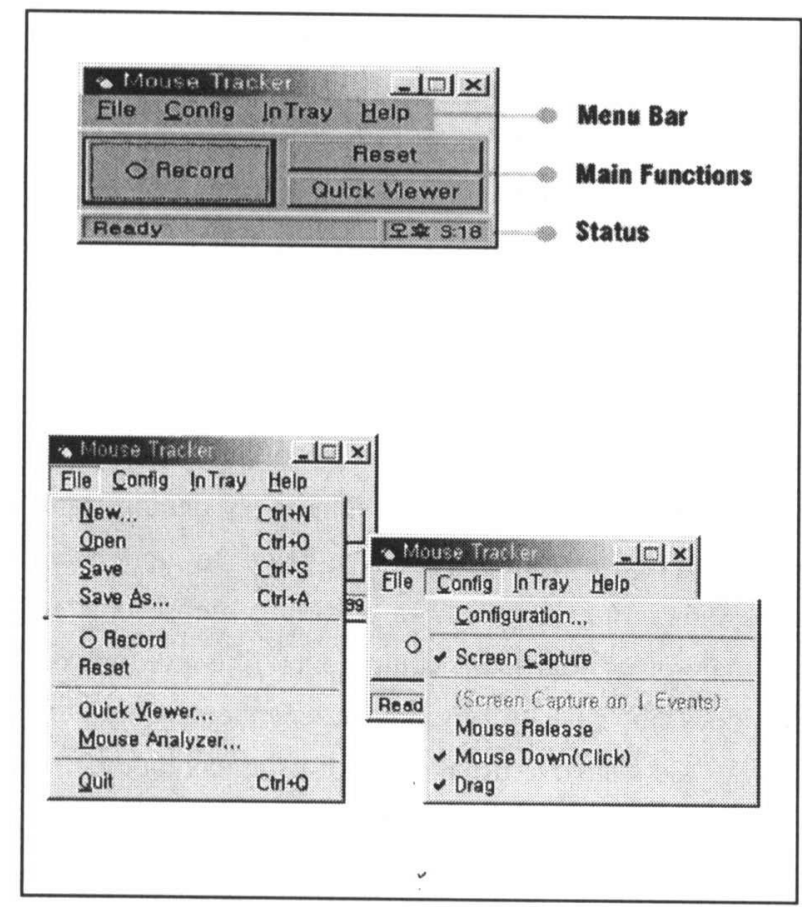

Figure 3: Menu Window for Mouse Tracker and Visualization of Operational Path of Mouse

The types of data Mouse Tracker saves include path of cursor movement, mouse operation point (click, drag, scroll), time duration between two clicks, and screen images. (Shown in the Figure 4)

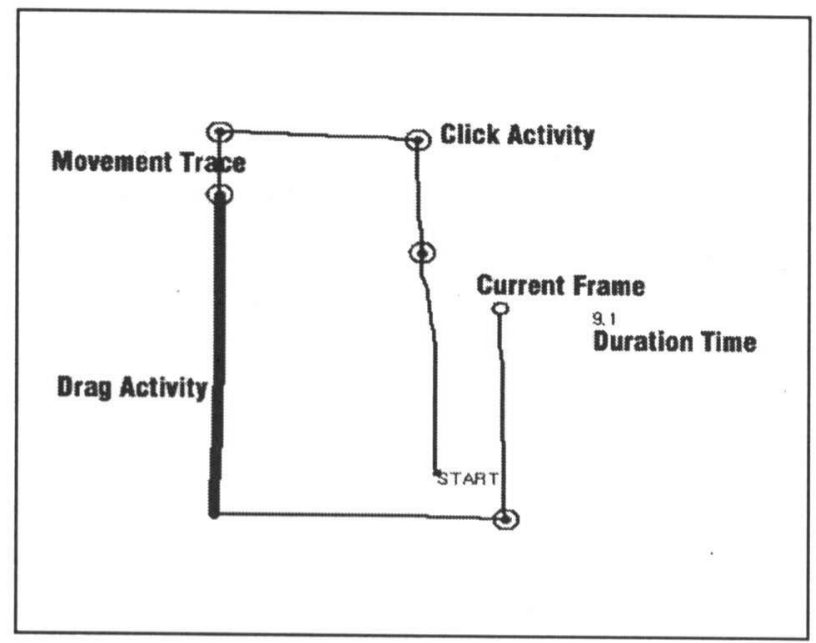

Figure 4: Types of Data Recorded and Its Visualization

Once record is over, designer can see visually displayed data of the entire user's mouse traces in various options in the mode of 'Quick Viewer' shown in Figure 5. As shown in the Figure, Quick Viewer has interface like VCR so that user can replay his interaction by pressing 'Play' button. Using this function makes possible for designer to understand how user navigates around web. During play mode, designer can stop whenever necessary for further detail analysis. Also users can set diverse option of viewing such as controlling interval, setting up starting and ending point, or filtering out specific mouse operations. All the functions are summarized in detail in Table 2.

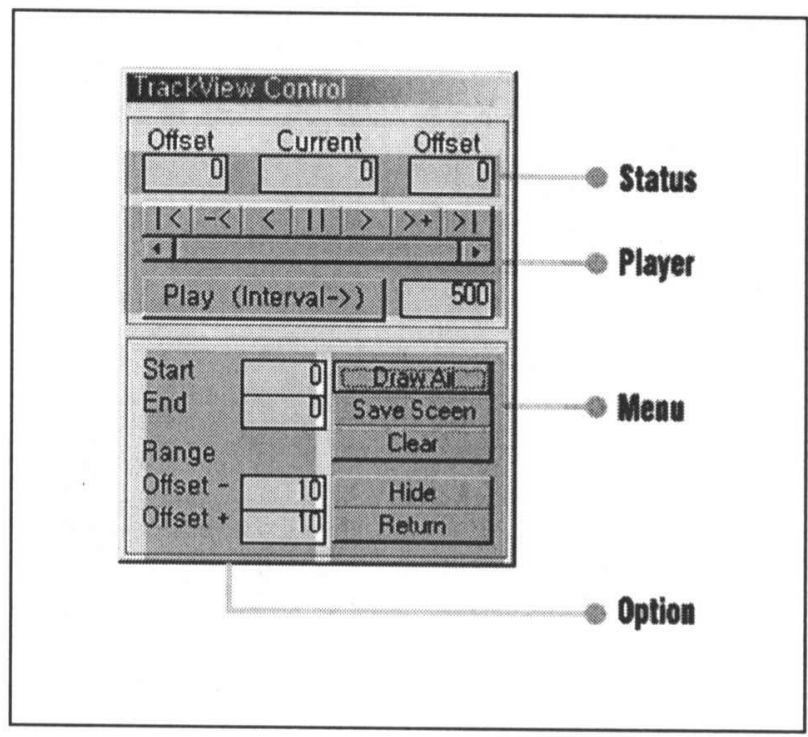

Figure 5: Composition of Quick Viewer

Table 2: Summary of Functions in Quick Viewer

\begin{tabular}{l|l|l}
\hline Types & Menu & Functions \\
\hline \multirow{4}{*}{ Status } & Offset & Frame \# of Offset \\
\cline { 2 - 3 } & Current & Current frame \# \\
\hline \multirow{4}{*}{ Player } & $\langle>|$ & Move to Start \& End \\
\cline { 2 - 3 } & $-<>+$ & Backward/Forward by Frames \\
\cline { 2 - 3 } & $<>$ & Backward/Forward by 1 Frame \\
\cline { 2 - 3 } & Play (Interval) & To start play (playing speed) \\
\hline \multirow{4}{*}{ Menu } & Draw All & Show entire paths \\
\cline { 2 - 3 } & Save Screen & Saving image as shown \\
\cline { 2 - 3 } & Clear & Clear out all the paths \\
\cline { 2 - 3 } & Hide & Hide Quick Viewer \\
\cline { 2 - 3 } & Return & Return to Mouse Tracker \\
\hline \multirow{5}{*}{ Option } & Start, End & Set the range of view \\
\cline { 2 - 3 } & Range Offset -+ & Set the range of Offset \\
\hline
\end{tabular}

The sample screen of 'Draw All' is shown in the Figure 6. As shown in the Figure 5, it is clearly shown where user started and ended, clicked, moved, and how long it took for each movement. By doing this, user can visually grasp user's overall Kansei pattern. If user wants to display only some specific range of mouse interaction, he also can set up the range. 


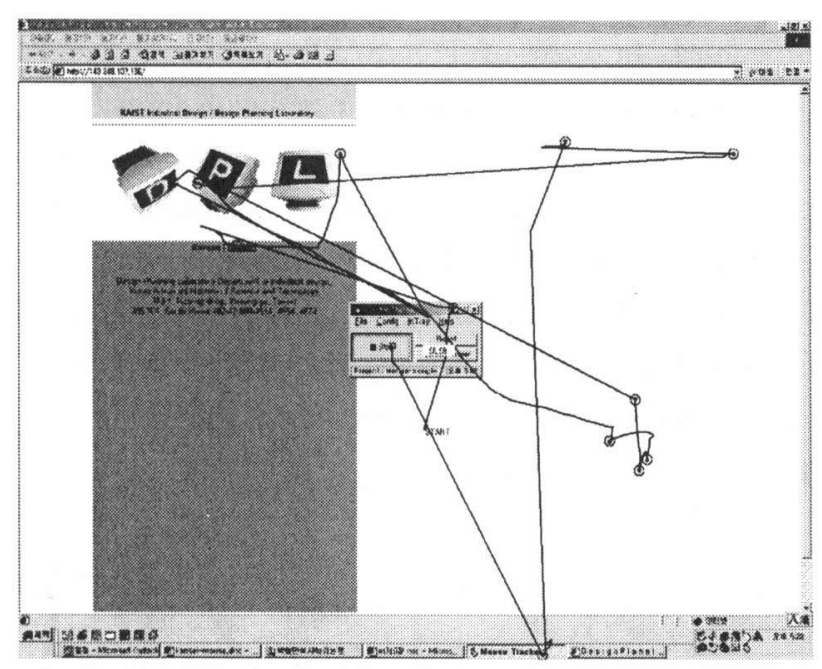

Figure 6: Sample Screen of Mouse Path Displayed on Web Page

User also can play within some range of frame so that he can see his operational paths with web pages he moved around. For example, Figure 7 shows that user moved three different pages.

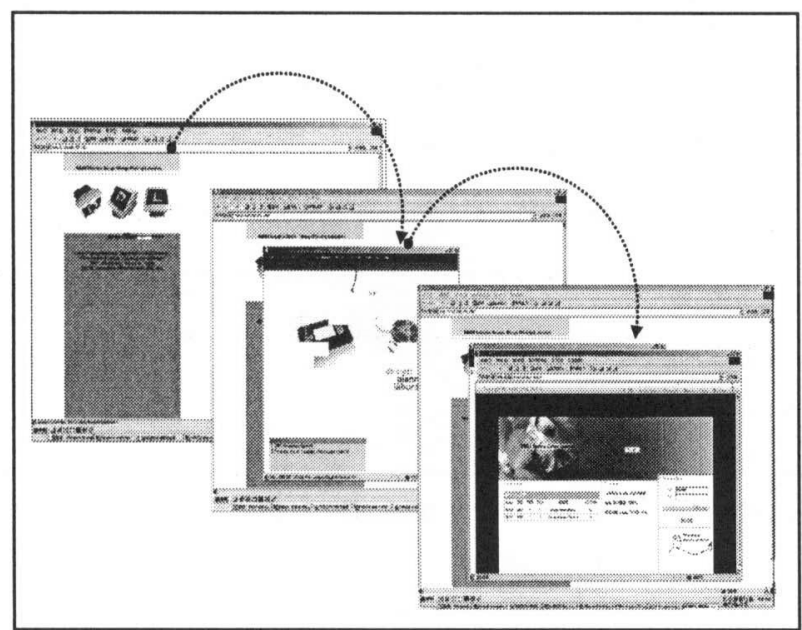

Figure 7: Saved Images of Web Pages User Moved Around

\subsection{Mouse Analyzer}

Once mouse tracking is done, user can further move to 'Mouse Analyzer' for more detail analysis. Mouse Analyzer allows user to conduct various analyses particularly in quantitative mode. Mouse Analyzer shown in Figure 8 consists of different sections: 'Viewer' for viewing specific web page with mouse path displayed on, 'Event Table' for listing up all data of time of clicking or activities, 'Word Palette' for making some notes on data, and 'Controller' for setting up diverse options. User can easily switch the sub-windows so that he can have more simultaneous and integrated analysis.

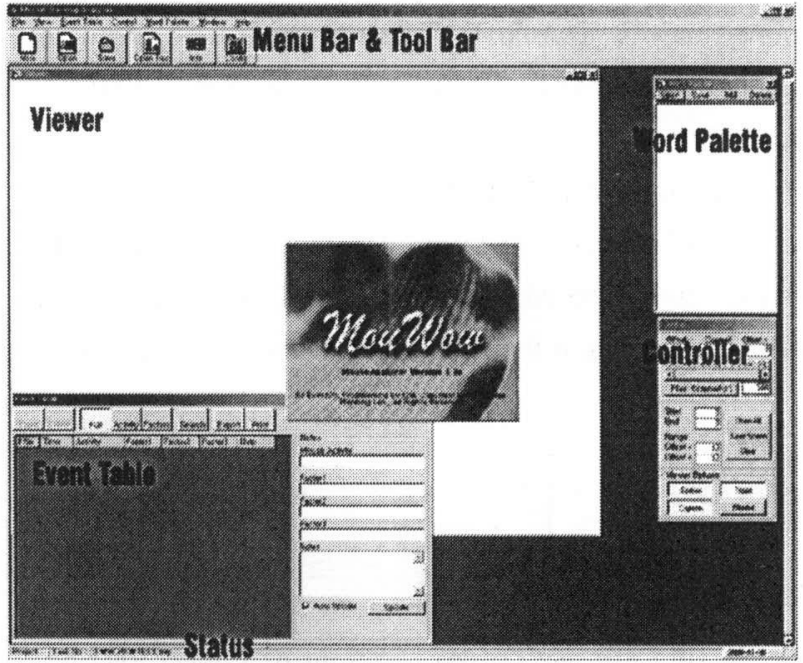

Figure 8: Structure of Mouse Analyzer

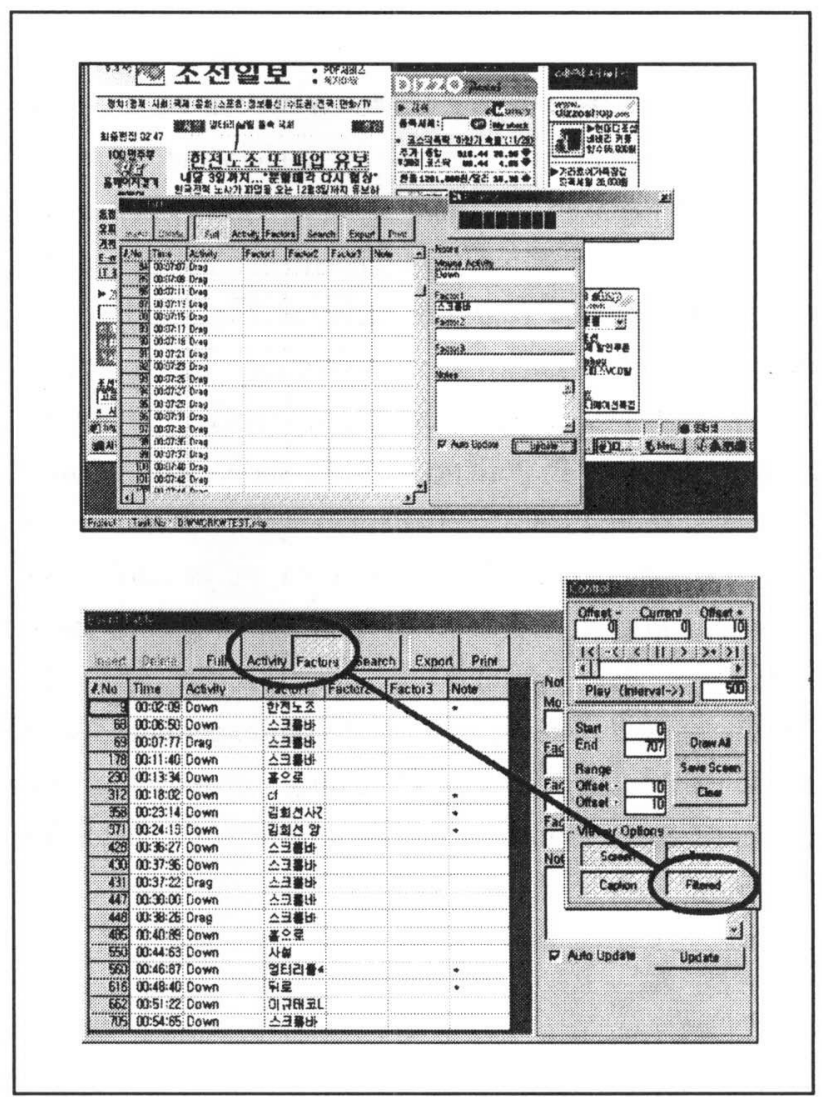

Figure 9: Sample Screen of Mouse Analyzer and Event Table

After loading the file saved form Mouse Tracker, all $\log$ data is listed up in Event Table like shown in Figure 8 (the event table is shown in more detail in the bottom part of Figure 9). User can select specific data by clicking a cell in event table, and then selected web page is displayed in Viewer. User can make some notes on it using Word Palette. User can easily sorted out specific types of data, for example, 'all the mouse operation taken 
more than 10 seconds' by using Controller. With this function, user can find some pattern of user's interaction behavior.

User is also able to have different options to view images of mouse tracking: dot mode of only clicked points; line mode without web page; full mode with web page like shown in the following Figure 10.

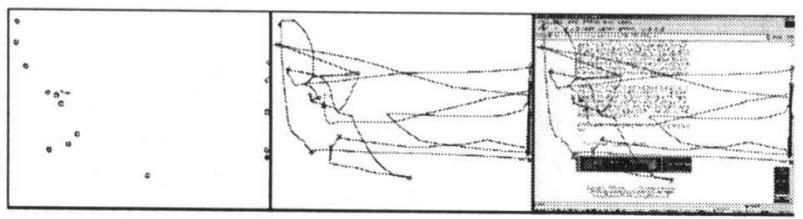

Figure 10: Different Display Options of Viewer Section

\section{APPLICATION OF TOOL}

The mouse tracking tool, MouWow can be used for various practical applications for understanding user's Kanse $i$ on the web. At first, it can reveal the pattern of user's perceptive Kansei by visualizing all the user's navigations around web. For example, Figure 11 shows different Kansei patterns of navigations between first comer (right) and experienced user (left) in shopping mall web site. Experienced user shows his Kansei pattern scattered with consistent density, which means that he scans through rather than stays and pays attentions in certain area while first comer spends some time and stays around in the specific area for familiarizing the web site. In addition, experienced user shows the pattern of natural surfing whereas the first comer concentrated on task-oriented behavior (purchasing some goods).

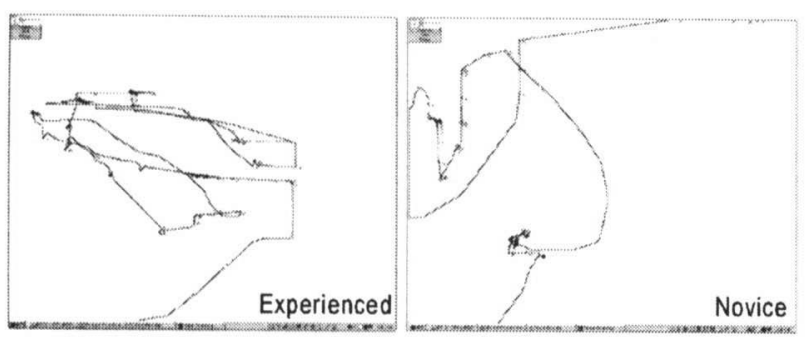

Figure 11: Comparison between Experienced and Novice User in the Perceptive Kansei Pattern

It can be also used as usability testing tool to identify the problem using the web. For instance, Figure 12 shows the comparison between correct path (left) and user's actual path (right). Comparison of these two patterns can effectively reveal where user experienced difficulties. In case of Figure 12, user shows quite different patterns of behavior compared with correct path, which reveals bottlenecks of interface (parts inside of red rectangular). Actual path of user also demonstrates that user moves frequently scroll up and down (note that thick drag line in the right side). It implies that the web interface does not give user any clear affordance of the web structure so that user kept moving up and down.
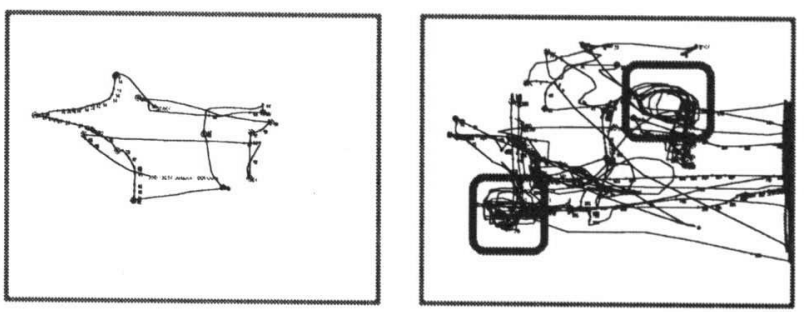

Figure 12: Comparison between Correct Path and User's Actual Path

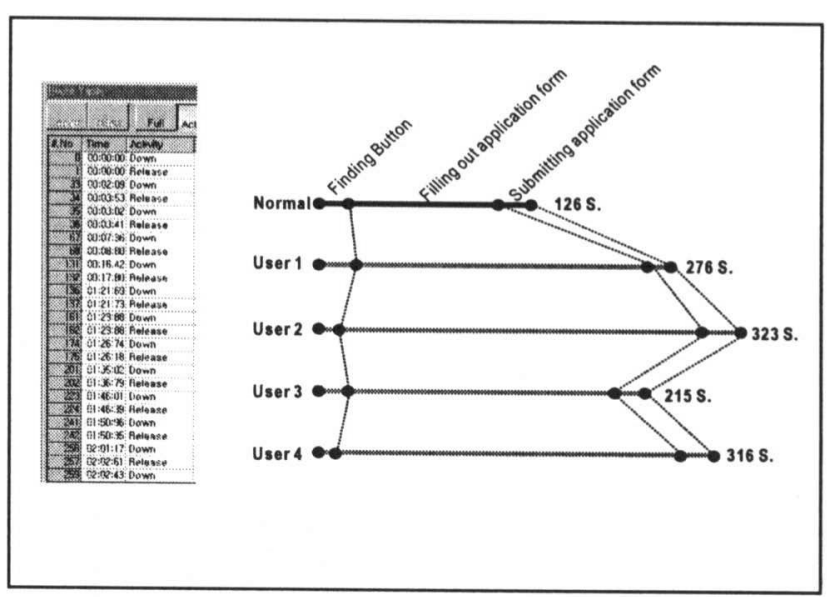

Figure 13: Event Table and Its Time Bar for Task Performance

Data of mouse tracking can be easily converted into other type of data for usability testing. For example, the quantitative data of event table can be transformed into diagram which can reveal how long it takes in the subtasks. Left side of Figure 13 shows the part of event table with its time data and right side of the Figure 13 shows the diagram of time taken in each sub-task. The diagram compares normal situation with actual performance done by users, so that a researcher can easily grasp in what sub-task user had most difficulties. For example, the task to given to users was to "get the ID" from a portal site. The task consists of "finding out ID application button and clicking", "filling out application form", and "submitting application form". The diagram clearly shows that users experienced most difficulties in filling 
out application form while they don't have any problems to find out ID application button and submitting application.

Another possible application of mouse tracking is to conduct more integrated analysis with actual user's behavior such as facial expression, body gesture, or protocol data. This type of analysis can reveal even more meaningful information regarding user's Kansei. Figure 14 shows some example of this type of application. As shown in the Figure, video clip of user's actual behavior is shown with the record of user's mouse track in parallel.

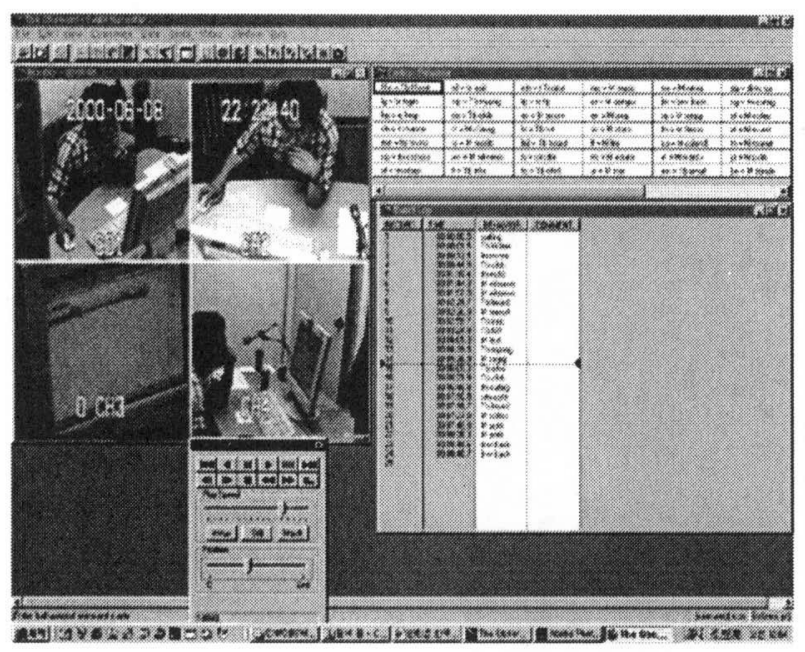

Figure 14: Parallel Analysis of Mouse Tracking and User's Actual Behavior

Besides of these four types of applications, there can be many other creative applications. For example, all the data can be exported to other software such as EXCEL or SPSS for further statistical analysis. For example, all the quantitative data in time \& event table can be exported and analyzed for 'Lag Sequential Analysis'. The Lag Sequential Analysis technique calculates the frequency of transition between pairs of events within a certain lag in a time series. Based on the frequency of transition between one mouse click to others, a researcher can get invaluable information of the probability of transition between them. This transition probabilities can be used to analyze the temporal structure of the user's navigational behavior in the web. [7] Or users can be segmented by their behaviors using patterns of mouse tracking.

The application of mouse tracking is not necessarily limited for the use of web only. It can be used for other digital information appliances such as mobile phone or PDA. Nowadays, digital information appliances go through usability testing by computer-simulated prototype. In other words, users perform tasks of computer-simulated product in the computer. In that case, users use mouse to perform task of product. Mouse track users left can be effectively used for understanding how user operates the product.

\section{CONCLUSION AND FURTHER WORKS}

The study showed the possibility of using patterns of mouse operation as understanding user's Kansei on web site. Particularly understanding user's Kansei in the use of web is quite difficult compared with other types of physical product because user does not show any concrete behavior. Some efficient tool needs to be developed which can reveal user's preference, interests, paths, or difficulties regarding web interface.

Diverse existing methods for understanding user's Kansei on web were reviewed and major difficulties were found. They include the ease of manipulation, high cost, unnatural operation, and difficulty to identify user's natural Kansei. In order to solve these problems, the actual mouse tracking software, MouWow was developed and demonstrated. User can record all his navigational paths over the web and visually display them with various options. In addition, user can analyze all the patterns in detail by annotating, filtering, or note taking. The study also showed various applications of the tool: understanding perceptive Kansei pattern; identifying interface problems in usability testing; understanding users integrated behavior by combining data of user's gesture, protocol data, or facial expression. It is possible to explore many other types of creative application of the tool.

However, the current tool is still in prototyping stage and needs to be further developed. At first, the movement of mouse does not necessary reflect user's preference and interest. Movement of mouse is depending on user's personal habit. Some user moves mouse more frequently pointing his interests while some other user only uses mouse for clicking and remains mouse on the scroll bar while not using. Therefore, in order for mouse tracking to reflect user's Kansei more correctly, researcher should always ask user to move his mouse according to his interest or eyesight, which may give psychological burden to user. Before starting experiment, user needs to have warming up session so that he can familiarize himself for mouse tracking. Secondly, MouWow should 
be further improved in terms of system stability, memory efficiency, and integrating some more functions.

If many types of data are accumulated for long time, it can be used as useful database. The data can be further analyzed in many different points of views: relationship between mouse pattern and cultural groups or comparison of mouse operation with different types of web.

\section{REFERENCE}

1. A.Harada; On the Definition of Kansei, Report of Modeling the Evaluation Structure of KANSEI; 22 (1998).

2. N.Jacob; Designing Web Usability: The Practice of Simplicity; New Riders, Indianapolis (2000).

3. Diamond Bullet Design; Usability Methods, [Online] Ann Arbor USA: Diamond Bullet Design. Available from URL: http://www.usabilityfirst.com/ methods/index.txl [Accessed 2001 April 15] (2001)

4. S.Marshall et al; How Do Your Users Really Use Your Site? Case Study of Eye Tracking for AT\&T, An ARF Week of Workshops Event; 29-36 (2000).

5. Eye Tracking Inc.; Web Site and Software Testing, [Online] San Diego USA: Eye Tracking Inc. Available from URL: http://www.eyetracking.com/ flash/index.htm [Accessed 2001 April 20] (2001)

6. D.ProBarker, M.S.Carey, R.G.Taylor; Factors Underlying Mouse Pointing Performance, Contemporary Ergonomics; 359-364 (1990).

7. L.Noldus, A.Kwint, W. Hove, R.Derix; Software tools for collection and analysis of observational data. In; H.J.Bullinger, J.Ziegler, J. (Ed.) Human-Computer Interaction: Communication, Cooperation and Application Design; 8th Int. Conf. on HumanComputer Interaction, Vol. 2, 1114-1118 (1999). 